\title{
Are long gamma-ray bursts biased tracers of star formation? Clues from the host galaxies of the Swift/BAT6 complete sample of LGRBs
}

\section{Stellar mass at $z<1^{\star}$}

\author{
S. D. Vergani ${ }^{1,2}$, R. Salvaterra ${ }^{3}$, J. Japelj ${ }^{4}$, E. Le Floc'h ${ }^{5}$, P. D'Avanzo ${ }^{2}$, A. Fernandez-Soto ${ }^{6,7}$, T. Krühler ${ }^{8}$,
} A. Melandri ${ }^{2}$, S. Boissier ${ }^{9}$, S. Covino ${ }^{2}$, M. Puech ${ }^{1}$, J. Greiner ${ }^{10}$, L. K. Hunt ${ }^{11}$, D. Perley ${ }^{12}$, P. Petitjean ${ }^{13}$, T. Vinci ${ }^{14}$, F. Hammer ${ }^{1}$, A. Levan ${ }^{15}$, F. Mannucci ${ }^{11}$, S. Campana ${ }^{2}$, H. Flores ${ }^{1}$,

A. Gomboc ${ }^{4}$, and G. Tagliaferri ${ }^{2}$

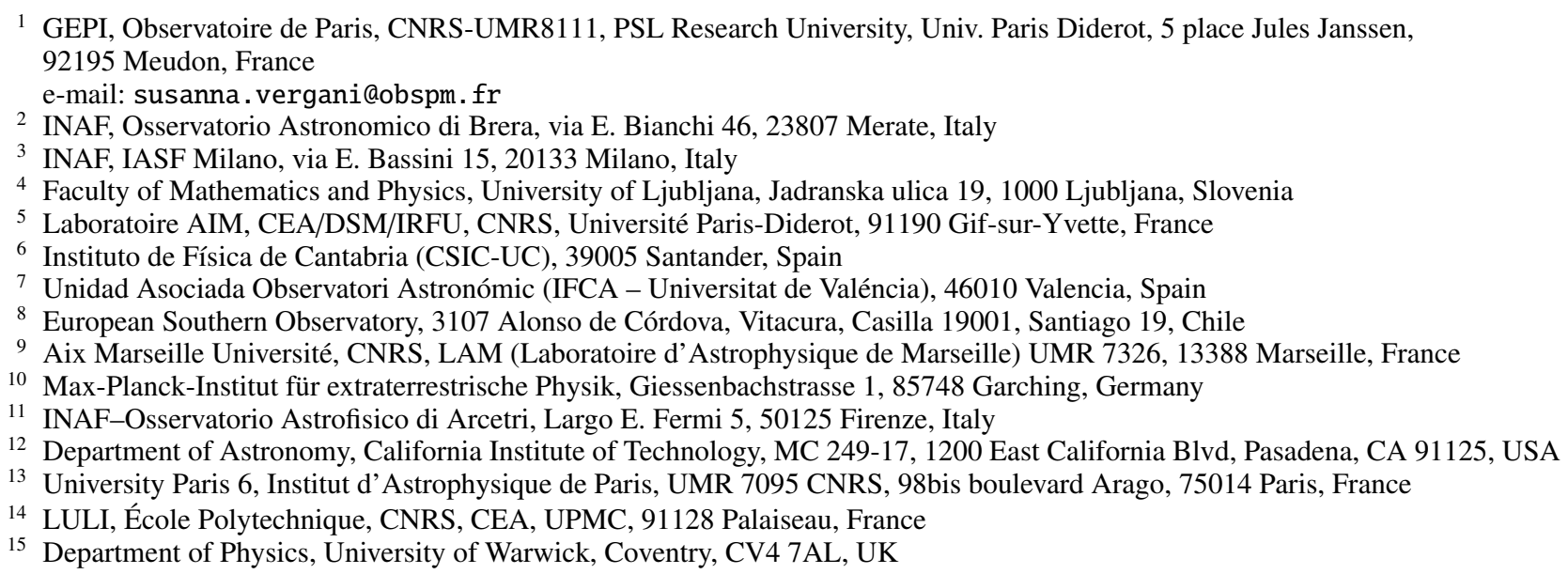

Received 17 September 2014 / Accepted 4 June 2015

\section{ABSTRACT}

\begin{abstract}
Aims. Long gamma-ray bursts (LGRBs) are associated with massive stars and are therefore linked to star formation. However, the conditions needed for the progenitor stars to produce LGRBs can affect the relation between the LGRB rate and star formation. By using the power of a complete LGRB sample, our long-term aim is to understand whether such a bias exists and, if it does, what its origin is.

Methods. To reach our goal we use the Swift/BAT6 complete sample of LGRBs. In this first paper, we build the spectral energy distribution (SED) of the $14 z<1$ host galaxies of the BAT6 LGRB sample and determine their stellar masses $\left(M_{\star}\right)$ from SED fitting. To investigate the presence of a bias in the LGRB-star formation relation we compare the stellar mass distribution of the LGRB host galaxies (i) with star-forming galaxies observed in deep surveys (UltraVISTA) within the same redshift limit; (ii) with semi-analytical models of the $z<1$ star-forming galaxy population; and (iii) with dedicated numerical simulations of LGRB hosts having different metallicity thresholds for the progenitor star environment.

Results. We find that at $z<1$, LGRBs tend to avoid massive galaxies and are very powerful for selecting a population of faint low-mass star-forming galaxies, partly below the completeness limits of galaxy surveys. The stellar mass distribution of the hosts is not consistent with that of the UltraVISTA star-forming galaxies weighted by their star formation rate (SFR). This implies that, at least at $z<1$, LGRBs are not unbiased tracers of star formation. To make the two distributions consistent, a much steeper faint end of the mass function would be required or a very shallow SFR-mass relation for the low-mass galaxy population. The comparison with the GRB host galaxy simulations indicates that, to reproduce the stellar mass distribution, a metallicity threshold of the order of $Z_{\mathrm{th}}=0.3-0.5 Z_{\odot}$ is necessary to form a LGRB. Models without a metallicity threshold or with an extreme threshold of $Z_{\mathrm{th}}=0.1 Z_{\odot}$ are excluded at $z<1$. Under a very basic assumption, we estimate that the LGRB rate can directly trace the SFR starting from $z \sim 4$ and above.

Conclusions. GRB hosts at $z<1$ have lower luminosities and stellar masses than expected if LGRBs were unbiased star formation tracers. The use of the Swift/BAT6 complete sample keeps this result from being affected by possible biases that could have influenced past results based on incomplete samples. The preference for low metallicities $\left(Z \lesssim 0.5 Z_{\odot}\right)$ inferred by the comparison with the simulations can be a consequence of the particular conditions needed for the progenitor star to produce a GRB.
\end{abstract}

Key words. gamma-ray burst: general - galaxies: star formation - galaxies: photometry

* Appendix A is available in electronic form at http://www. aanda.org 


\section{Introduction}

In recent years, great progress in understanding cosmic star formation has come through studying populations of star-forming galaxies selected through different techniques. However, each sample suffers from uncertainties arising from dust extinction, flux-limited samples, and incomplete redshift determinations. Here is where long gamma-ray bursts (LGRBs, prompt emission duration longer than $2 \mathrm{~s}$ ) can play an important role.

GRBs are short, intense bursts of gamma-rays that during seconds to min outshine all other sources of gamma-ray emission in the sky. Following the prompt gamma-ray emission an "afterglow" emission in the range from X-ray to radio wavelengths persists up to months after the initial burst. Uniquely, GRBs allow galaxies to be selected independently of their brightnesses at any wavelength. The association of LGRBs with the explosion of broad-line type Ic SNe (see Hjorth \& Bloom 2011, for a review), hence with the death of massive stars, makes this class of GRBs a unique tool for understand the evolution of star formation and galaxies, complementary to current galaxy surveys, up to the highest redshifts $(z=8.2$; Salvaterra et al. 2009; Tanvir et al. 2009; $z \sim 9$; Cucchiara et al. 2011).

Indeed, the observations of the $z>5$ LGRB host galaxies resulted in very deep limits on their magnitudes, which are as faint as 30.3(AB) in $H$-band (Tanvir et al. 2012; Basa et al. 2012). This is consistent with the results of numerical simulations showing that LGRBs pinpoint star-forming galaxies belonging to the faint end of the luminosity function. Such galaxies form the bulk of the first galaxies population that should significantly contribute to re-ionisation (Salvaterra et al. 2011).

Several studies have targeted LGRB host galaxies (e.g. Le Floc'h et al. 2003, 2006; Christensen et al. 2004; Fruchter et al. 2006; Savaglio et al. 2009; Castro Cerón et al. 2010; Svensson et al. 2010; Krühler et al. 2015). LGRB hosts at $z<1$ are in general found to be sub-luminous, blue, moderately star-forming $\left(S F R \sim 1 M_{\odot} \mathrm{yr}^{-1}\right)$, and with stellar masses $M_{\star} \sim 1-5 \times 10^{9} M_{\odot}$ (but see e.g. Krühler et al. 2011, 2015). The observed luminosity distribution of LGRB hosts is broad, indicating that a considerable amount of star formation takes place in low luminosity galaxies. Han et al. (2010), Levesque et al. (2010), Graham \& Fruchter (2013), Wang \& Dai (2014) show that LGRB host galaxies (at least at $z<1$ ) have a lower metallicity than do other galaxies at similar masses, tracing an environment that is less chemically evolved than the galaxies selected by other techniques. On the other hand, at $z<1$, LGRB hosts are found to be consistent with the fundamental metallicity relation (FMR; Mannucci et al. 2010, 2011), disfavouring the very low metallicity thresholds expected from the LGRB theoretical models, which would have a strong impact on the properties of the galaxy population hosting the LGRB event.

The host galaxies of LGRBs have also been studied by numerical simulations or semi-analytical models (e.g.: Nuza et al. 2007; Chisari et al. 2010; Mao 2010; Artale et al. 2011; Niino et al. 2011; Elliott et al. 2012; Trenti et al. 2015). Campisi et al. (2011) studied the mass-metallicity relation and the FMR of LGRB host galaxies comparing 18 LGRB host galaxies with a catalogue of simulated galaxies constructed combining highresolution $N$-body simulations with a semi-analytic model of galaxy formation. They found that a very low-metallicity threshold $\left(Z<0.3 Z_{\odot}\right)$ is not required to reproduce either relations, explaining the observed low metallicities as a consequence of LGRBs exploding in low-mass and strongly star-forming galaxies.
With a new approach, Boissier et al. (2013) tried to determine the GRB efficiency (i.e. the GRB rate - SFR ratio) and its causes more quantitatively by studying the distribution of LGRB host properties with respect to the star-forming galaxy population, so as to still be able to properly use LGRBs as starformation rate (SFR) tracers.

The picture seems to become more complex when the host galaxies of the so-called dark LGRBs (see Jakobsson et al. 2004; van der Horst et al. 2009, for a definition) are considered, and when the studies are extended to higher redshift and/or longer wavelength domains. Krühler et al. (2011), Perley et al. (2013), and Hunt et al. (2014) found that on average dark LGRBs extend the LGRB host galaxy population to more massive galaxies with a higher SFR. For some of the few host galaxies detected in the far-infrared, sub-millimetric, and radio domains (e.g. Berger et al. 2003; Michałowski et al. 2008, 2012; Hunt et al. 2014; Schady et al. 2014; Symeonidis et al. 2014; Perley et al. 2015b), very high SFR values have been found, up to $10^{3} M_{\odot} \mathrm{yr}^{-1}$.

All the observational studies cited above are based on different, heterogeneous samples of LGRB host galaxies, which are far from being complete and which are likely to be biased, therefore they can obtain different results on LGRB host galaxies properties (e.g. Perley et al. 2013 and Hunt et al. 2014 reach different conclusions about GRBs as SFR tracers). Nonetheless, there is some agreement on the fact that, at $z<1$, LGRBs seem to prefer lower mass systems relative to a purely SFR-selected galaxy sample. To have an unbiased picture of the properties of LGRB hosts, complete samples of LGRBs are necessary.

Three complete samples of GRBs have become available between 2011 and 2012: the GROND ${ }^{1}$ (Greiner et al. 2008) sample (Greiner et al. 2011), the BAT6 sample presented in Salvaterra et al. (2012), and the TOUGH sample (Hjorth et al. 2012). Furthermore, Perley et al. (2015a,c) present a study based on Spitzer observation of the unbiased Swift Host Galaxy Legacy (SHOALS) Survey. In this paper we focus on the BAT6 sample.

The BAT6 LGRBs are selected only on the basis of the brightness of their gamma-ray prompt emission (Swift/BAT peak flux $P \geq 2.6 \mathrm{ph} \mathrm{s}^{-1} \mathrm{~cm}^{-2}$ ), and on favourable observing conditions (practically not introducing any bias, see Jakobsson et al. 2006). The cut on the brightness of the gamma-ray emission is needed to guarantee the completeness. In fact, getting closer to the BAT detection threshold, Swift starts to miss some GRBs. The sample consists of 58 LGRBs, and it is complete in redshift at $95 \%$. We note that the detection of the optical afterglow is not required in the construction of the BAT6 sample, therefore dark bursts are represented well within the sample (see Melandri et al. 2012). This sample offers the unique opportunity to study the population of LGRBs in a statistically unbiased way. In particular, as the brightness of the gamma-ray prompt emission is the only burst-depending parameter for selecting the LGRB sample, the BAT6 allows the selection of a complete sample of LGRB host galaxies independently of their fluxes or colours.

Our project is to study the properties of the host galaxies of the $z<1$ LGRBs of the BAT6 with the aim of investigating the relation between LGRB and star formation. In this paper we present the study of the stellar masses. We restrict ourselves to LGRBs at $z<1$ because i) of the wealth of data available in this redshift range; ii) of the larger differences expected in this redshift range on theoretical grounds between the LGRB hosts and the normal field populations; and iii) of the range where the study of the properties of the galaxies can be carried out by optical/near-infrared instruments. It is not possible

http://www .mpe.mpg.de/ jcg/GROND/ 
at the moment to maintain the same completeness level in the galaxy property determination at higher redshift for our sample.

In Sect. 2 we present the host galaxy sample and the data used. The K luminosity and the stellar masses are studied in Sect. 3 and compared to those of the UltraVISTA survey in Sect. 4. Application of the Boissier's method to our data is presented in Sect. 5, whereas in Sect. 6 we compare the stellar mass distribution with the LGRB host galaxy simulations. Discussion and conclusions are carried out in Sects. 7 and 8, respectively.

\section{The sample of LGRB host galaxies}

We selected the $z<1$ LGRB in the BAT6 sample, corresponding to 14 objects. To determine the stellar masses of the host galaxies of the selected objects, we collected the photometry published in the literature and all the available photometric data. In addition we obtained new data to complete the spectral energy distributions (SEDs) when needed. We also checked for Spitzer observations and found that 12 of 14 of the hosts have IRAC1 or IRAC2 observations. More than half of the hosts in the sample are detected, and upper limits can be obtained for the remaining observed hosts, allowing the coverage of the rest-frame $K$-band.

All the magnitudes collected for each galaxies can be found in Table 1. We give here a brief description of the newly determined photometry, both from our new observational programmes and from the public unpublished data. These magnitudes were corrected for the Milky Way extinction according to the values of Schlafly \& Finkbeiner (2011), assuming RV = 3.08. More details on all the photometry used for each host galaxy can be found in Appendix A.

\subsection{New GROND data and photometry}

We obtained GROND data that was not published before for the host galaxies of GRB 080916A, GRB 081007 and GRB 091018, in all the GROND 7 filters. All data were calibrated against the SDSS or 2MASS. Standard aperture photometry was used to derive the host flux with an appropriate aperture correction to get total fluxes. The magnitudes are based on a stack of different dithered images (12-14 in griz, 360-480 in JHK) and were only those images that fulfil a certain set of criteria in terms image quality are used, as explained in details in Krühler et al. (2011). The errors include both the statistical errors of the detection and the error in the photometric calibration.

\subsection{New GTC data and photometry}

GTC data were obtained with OSIRIS for the host galaxies of GRB 050525 ( $g$ and $i$-bands) and GRB $071112 \mathrm{C}$ ( $z$-band) under the programme GTC31-13B (PI: A. Fernandez-Soto). The data were reduced using standard procedures with tools provided by the ESO-Eclipse package (Devillard 1997). Photometry was carried out with SExtractor (v2.8.6, Bertin \& Arnouts 1996). Instrumental zero points were measured by observing standard stars SA112-805 and SA104-428 (Smith et al. 2002) in the case of $050525 \mathrm{~A}$ and $071112 \mathrm{C}$ hosts, respectively. Standard aperture photometry with an appropriate aperture correction was applied to derive the host galaxy magnitudes. Final errors include both statistical errors and the uncertainties of the photometric calibration.

\subsection{New VLT/HAWK-I data and photometry}

New VLT/HAWK-I data were obtained for the host galaxies of GRB 061021, GRB 091018, GRB 080916A (Programme ID 092.D-0305, PI: S. D. Vergani), and GRB 081007 (Programme ID 092.A-0231, PI: T. Kruhler). The data were obtained with the $J$ and $K$ filters for all the host galaxies except for GRB 091018 for which only the $K$ filter has been used.

HAWK-I images were reduced using the ESO HAWK-I pipeline $v 1.8 .12^{2}$ following the monolithic reduction cascade ${ }^{3}$, which includes background subtraction, flat field and illumination correction, sky subtraction, astrometric offset refinement, stacking of the images and distortion correction. Photometry was carried out with SExtractor (v2.8.6, Bertin \& Arnouts 1996). Standard aperture photometry with an appropriate aperture correction has been applied to derive the host galaxy magnitudes. If a host is not detected in the image, we report $3 \sigma$ upper limits. Magnitudes have been calibrated against several unsaturated 2MASS stars in the field of view in the science frames. Final errors include both statistical errors as well as the uncertainties of the photometric calibration.

\subsection{New TNG data and photometry}

New TNG photometry has been obtained for the hosts of GRB 080430 (OPTICON ID: OPT13B_71; PI S. D. Vergani; giz filters). We used also some public but unpublished data (programme ID: A22_TAC107, PI: D. Malesani), retrieved from the TNG archive ${ }^{4}$ for the host galaxies of GRB060912A ( $U B V I J$ filters), GRB061021 ( $B$ and $V$ filters), GRB071112C (program ID: A17_TAC9, PI: C. Guidorzi; I filter)

Image reduction was carried out following standard procedures: subtraction of an averaged bias frame and division by a normalised flat frame. The photometric calibration was achieved by observing Landolt standard fields. Aperture photometry was performed using the SExtractor package (Bertin \& Arnouts 1996). Astrometric solutions were computed against the USNO-B1.0 catalogue ${ }^{5}$.

\subsection{New Gemini photometry}

For the host galaxy of GRB $071112 \mathrm{C}$, we used public $R$-band Gemini data that has not been published previously, retrieved from the Gemini archive $^{6}$; program ID: GN-2008B-Q-112 PI: A. Levan). Image reduction was carried out following the standard procedures described in Sect. 2.4.

\subsection{New HST photometry}

We retrieved from the HST archive ${ }^{7}$ the F160W public HST data (not published before), for the host galaxies of GRB 071112C, GRB 080430, GRB 080916A, and GRB 081007 (programme ID: 12307, PI: A. Levan). HST/WFC3 observations were reduced in the standard HST fashion. Photometry was carried out with SExtractor (v2.8.6, Bertin \& Arnouts 1996) and calibrated with

\footnotetext{
2 http://www.eso.org/sci/software/pipelines/

$3 \mathrm{ftp}$ ://ftp.eso.org/pub/dfs/pipelines/hawki/ hawki-pipeline-manual-1.9.pdf

4 http://ia2.oats.inaf.it/index.php/tngarchive/tng

5 http://www.nofs .navy.mil/data/fhcpix

6 http://www2 .cadc-ccda.hia-iha.nrc-cnrc.gc.ca/en/ gsa/

7 https://archive.stsci.edu/hst/search.php
} 


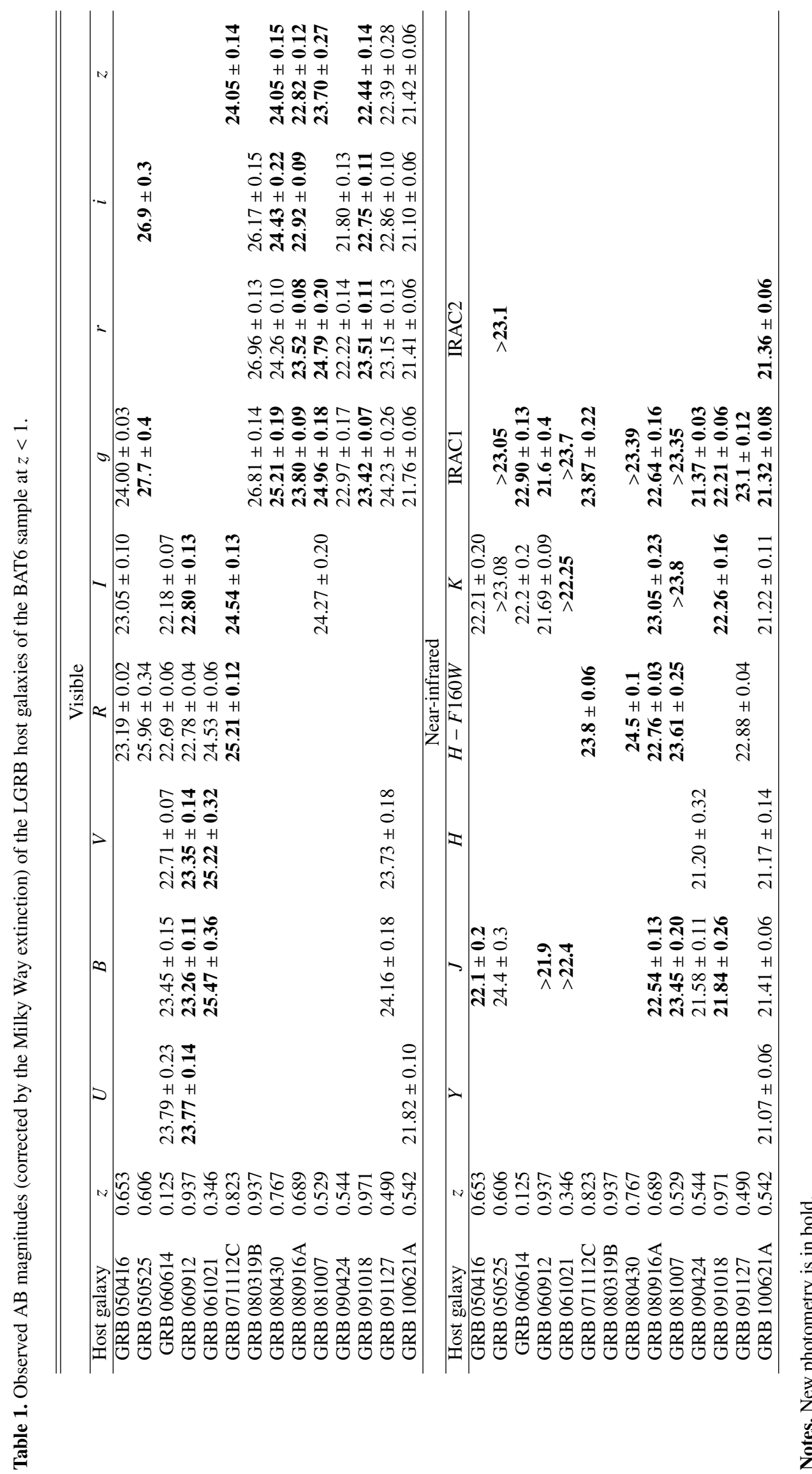


the official WFC3 instrument zero points ${ }^{8}$. Standard aperture photometry with an appropriate aperture correction has been applied to derive the host galaxy magnitudes.

\subsection{New Spitzer photometry}

We use public Spitzer data not previously published, retrieved from the Spitzer archive ${ }^{9}$, for all the host in the sample except for GRB 050416 and GRB 080319B. The data come from the different observing programmes of D. Perley (see the Appendix for details) except for the host of GRB 050525 (programme ID: 3653, PI: P.M. Garnavich). The IRAC fluxes were determined using the PBCD (post-basic calibrated data) and applying aperture photometry (1.9" radius) following the prescriptions of Sanders et al. (2007).

Given the lower spatial resolution of the IRAC data, special attention was devoted to addressing possible causes of confusion. Significant flux contamination and blending was found for only one object in our sample (GRB 060912A), which lies $\sim 11^{\prime \prime}$ away from a much brighter nearby galaxy. The contribution of the latter within the apertures used for the GRB host photometry (sky + source) was estimated pixel by pixel by fitting its surface brightness with a Sersic profile model, which was subsequently subtracted from the IRAC image before estimating the GRB host photometry.

\section{The host galaxy K luminosities and stellar masses}

We fitted the SED of the host galaxies with the code LePhare ${ }^{10}$ (Arnouts et al. 1999; Ilbert et al. 2006). We used the Stellar Population Synthesis (SPS) package developed by Bruzual \& Charlot (2003), with the BC03_m52 library. The metallicity is therefore fixed to $Z=0.008$ (i.e. $\sim 0.4 Z_{\odot}$ ). We assumed an universal IMF from Chabrier (2003) and an exponentially declining star formation history $S F R \propto \mathrm{e}^{-t / \tau}$, with a $\tau$ grid of $0.1,0.3$, $1,2,3,5,10,15$, and 30 Gyr. Dust extinction was applied to the templates using the Calzetti et al. (2000) law, with a $E_{(B-V)}$ grid going from 0.0 to 0.9 with a 0.05 step. The SED fitting plots and results for each host galaxy are reported in Fig. A.1 and Table A.1, respectively.

Two LGRB hosts (GRB 080319B and GRB 050525) do not have NIR detections or stringent upper limits, therefore their $\mathrm{K}$ luminosity (or stellar mass) is poorly costrained. We report in Table 2 the K luminosity $L_{\mathrm{K}}$ obtained from the SED fitting for each host galaxy. A determination using a $K$-corrected flux from the closest band has also been retrieved, giving similar results.

The stellar masses $\left(M_{\star}\right)$ have been obtained by the SED fitting described above and are reported in Table 2 . We verified that varying the input metallicity using the BC03_m62 library $\left(Z=0.02 \sim Z_{\odot}\right.$ ), or leaving the metallicity free to vary, the stellar mass values obtained are consistent within errors, not affecting significantly the final distribution. We also considered a Small Magellanic Cloud extinction law (Prevot et al. 1984). Again, the resulting stellar masses are consistent within the uncertainties. Only 5 of the 14 host galaxies had the stellar mass determined before (see Table 2). For all these cases, our results are consistent with the values obtained in previous works.

\footnotetext{
8 http://www.stsci.edu/hst/wfc3/phot_zp_lbn

9 http://irsa.ipac.caltech.edu/Missions/spitzer.html

10 http://www . cfht . hawaii . edu/ arnouts/LEPHARE/lephare. html
}

Table 2. Absolute $B$ magnitudes $\left(\mathrm{M}_{B}\right.$; from the SED fitting), $L_{\mathrm{K}}$, and stellar masses of the LGRB host galaxies of the BAT6 sample at $z<1$.

\begin{tabular}{|c|c|c|c|c|}
\hline Host galaxy & $z$ & $M_{B}$ & $\begin{array}{c}\log \left(L_{\mathrm{K}}\right) \\
{\left[\operatorname{erg~s}^{-1} \mathrm{~Hz}^{-1}\right]}\end{array}$ & $\begin{array}{l}\log \left(M_{\star}\right) \\
{\left[M_{\odot}\right]}\end{array}$ \\
\hline GRB $050416^{a}$ & 0.653 & -19.6 & 28.6 & $9.17_{-0.12}^{+0.12}$ \\
\hline GRB 050525 & 0.606 & -15.5 & 28.0 & $8.1_{-0.6}^{+0.6}$ \\
\hline GRB $060614^{a}$ & 0.125 & -15.7 & 27.2 & $8.09_{-0.17}^{+0.13}$ \\
\hline GRB 060912 & 0.937 & -20.8 & 29.2 & $9.23_{-0.07}^{+0.06}$ \\
\hline GRB 061021 & 0.346 & -16.3 & 27.6 & $8.5_{-0.5}^{+0.5}$ \\
\hline GRB 071112C & 0.823 & -18.5 & 28.3 & $8.89_{-0.18}^{+0.15}$ \\
\hline GRB $080319 B^{b}$ & 0.937 & -17.3 & 27.4 & $8.1_{-0.4}^{+0.4}$ \\
\hline GRB 080430 & 0.767 & -18.5 & 27.8 & $8.15_{-0.20}^{+0.12}$ \\
\hline GRB 080916A & 0.689 & -19.6 & 28.4 & $8.98_{-0.08}^{+0.07}$ \\
\hline GRB 081007 & 0.529 & -17.6 & 27.8 & $8.78_{-0.45}^{+0.08}$ \\
\hline GRB 090424 & 0.544 & -20.0 & 28.9 & $9.38_{-0.19}^{+0.17}$ \\
\hline GRB 091018 & 0.971 & -20.9 & 29.0 & $9.52_{-0.10}^{+0.08}$ \\
\hline GRB $091127^{c}$ & 0.490 & -18.7 & 28.1 & $8.67_{-0.07}^{+0.07}$ \\
\hline GRB $100621 \mathrm{~A}^{d}$ & 0.542 & -20.6 & 29.0 & $9.04_{-0.05}^{+0.06}$ \\
\hline
\end{tabular}

Notes. ${ }^{(a)} M_{\star}$ consistent with that reported in Savaglio et al. (2009); (b) $M_{\star}$ consistent with what is reported in Tanvir et al. (2010); ${ }^{(c)} M_{\star}$ consistent with that reported in Vergani et al. (2011); ${ }^{(d)} M_{\star}$ consistent with what is reported in Krühler et al. (2011).

Star-bursting galaxies have their light dominated by the youngest stellar populations. In relatively evolved galaxies undergoing a burst of star formation, this implies that the oldest populations can be difficult to detect since their light can be largely outshined by the youngest stars. This can result in large systematic errors that lead to underestimating their stellar mass when using a single stellar population SED fitting method (e.g. Pforr et al. 2012). To investigate the possible impact of such an uncertainty, we followed Papovich et al. (2001) and re-fitted each observed SED with the best-fit template to which an additional stellar population of old stars was added. For each galaxy, this old population was set as the SPSs with same parameters that the best-fit SED excepts the age, which was set to the age of the Universe at the observed redshift. In principle, this can constrain the maximum contribution of old populations within the photometric error bars (see Papovich et al. 2001, for details). We find a negligible contribution to the stellar mass (i.e. variations much smaller than the statistical uncertainty associated with the best-fit template) in all cases except GRB 071112C for which the stellar mass slightly increases to $\log M_{\star}=9.1 M_{\odot}$. This analysis confirms that LGRB hosts at $z<1$ are young starforming objects, which are dominated by young stars. To also obtain strict upper limits on the stellar masses of the hosts, we used the method by Bell et al. (2003) developed using bright local galaxies, assuming neither evolution in IMF nor a mass-tolight ratio.

\section{Comparison with star-forming galaxies}

To investigate the relation between LGRBs and SFR, we compared our LGRB host galaxies to the star-forming galaxies detected the UltraVISTA survey (McCracken et al. 2012; Ilbert et al. 2013; Muzzin et al. 2013). For this comparison we used the results obtained by the SED fitting described at the beginning of Sect. 3, because the SED fitting of the galaxies in the survey (see Ilbert et al. 2013) is performed in a similar way. 


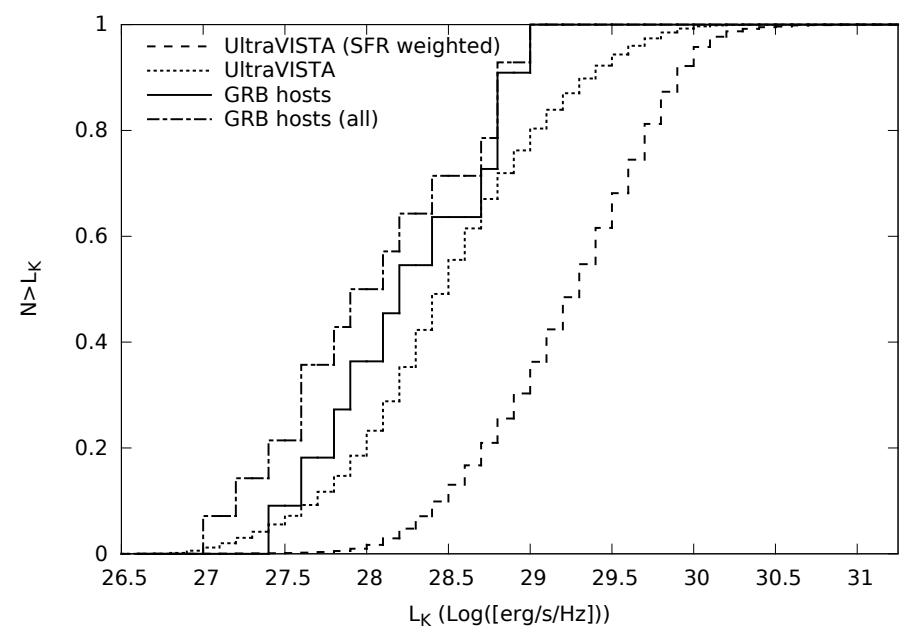

Fig. 1. Cumulative distribution of the K luminosity $\left(L_{\mathrm{K}}\right)$ of the starforming galaxies in the UltraVISTA survey at $z<1$ (dotted line) weighted by the SFR (dashed line) compared to that of the LGRB hosts in our sample (solid line: without the three LGRB host galaxies not complying with the UltraVISTA redshift and $K$-band magnitude cuts; dot-dashed line: whole sample).

\section{1. $L_{K}$}

In Fig. 1 we plot the (normalised) $L_{\mathrm{K}}$ cumulative distribution of the LGRB hosts and of the UltraVISTA star-forming galaxies at $z<1$. We used the UltraVISTA K luminosities and SFRs resulting from the SED fitting obtained by the same authors. The UltraVISTA survey has a cut by definition at $m_{\mathrm{K}}(\mathrm{AB})=$ 24. The SED fitting of the host galaxies of GRB 080319B and GRB 080430 indicates $K$-band magnitudes lower than this limit. The UltraVISTA results do not consider galaxies at $z<0.2$. GRB 060614 lies below this limit. In Fig. 1 we report the LGRB host $L_{\mathrm{K}}$ cumulative distribution with and without taking the host galaxies into account, and not complying with the survey cuts or having a poor SED (i.e. excluding 3 host galaxies).

Since LGRBs are the end product of some massive stars, the probability of hosting a LGRB is proportional to the SFR of a galaxy, at first approximation. Therefore, when comparing the hosts with the UltraVISTA galaxies, we should weight the K luminosity of the latter by their SFR. We do this by putting the sum of the SFR values in each bin (resulting from the survey SED fitting) of each galaxy falling in that bin, instead of simply adding the number of galaxies.

Because of the fast increase in SFR with $z$, the UltraVISTA SFR-weighted galaxy distribution at $0.2<z<1.0$ might be dominated by galaxies close to $z \sim 1$, hence having higher masses due to the UltraVISTA mass completeness limit (Ilbert et al. 2013), whereas the GRB lies at a slightly lower mean redshift $(z=0.64)$. This could have some effect on the comparison between the $L_{\mathrm{K}}$ cumulative distribution of the LGRB hosts and of the UltraVISTA star-forming galaxies. Nonetheless, we verified that this effect is small: when considering only the UltraVISTA galaxies at $0.5<z<0.8$ (i.e. around the average redshift of the GRBs in our sample), we obtain a similar SFRweighted distribution and discrepancy from that of LGRB host galaxies.

Considering $L_{\mathrm{K}}$ as a proxy for the stellar mass, from Fig. 1 we can already infer that the stellar mass distribution of LGRB hosts is not consistent with that of the star-forming galaxies at $z<1$ weighted by they SFR, implying that at $z<1$ LGRBs are not unbiased tracers of the star formation.

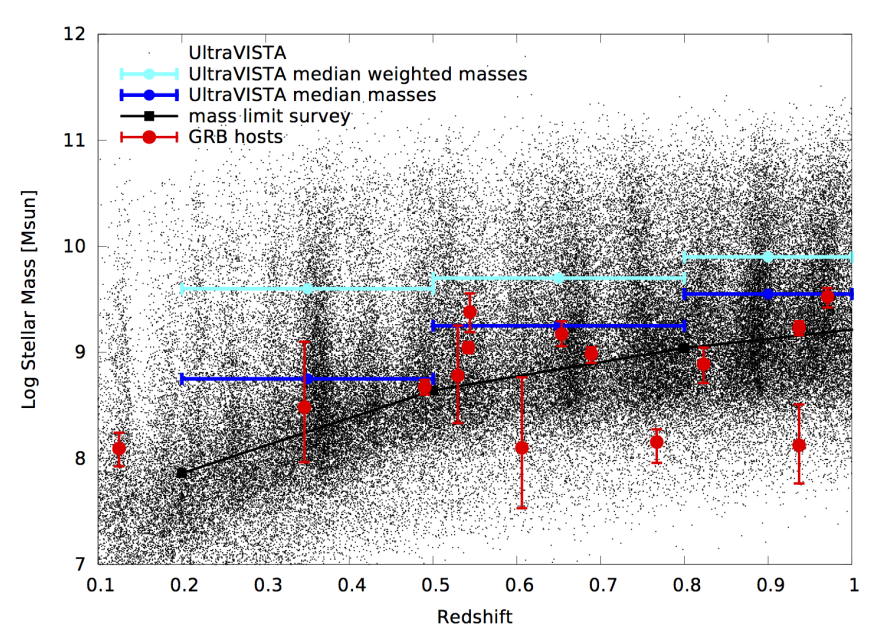

Fig. 2. Stellar masses as a function of redshift for the LGRB host galaxies (red and green dots), overplotted on those of the UltraVISTA survey. The black solid line shows the mass completeness of the survey. The blue lines refer to the survey median stellar masses, whereas the cyan lines refer to the same quantity weighted by the galaxy SFR.

\subsection{Stellar mass}

In Fig. 2, we plot the stellar masses as a function of redshift for the LGRB host galaxies and for the star-forming galaxies of the UltraVISTA survey weighted by their SFR. We considered the mass completeness limits of Ilbert et al. (2013), also reported in the figure. To weight the galaxy stellar masses by their SFR, we proceeded as described in Sect. 4.1. As above, we used the SFR values obtained from the $S E D$ fitting $\left(S_{S F R}\right)$. At this redshift range, the SFR $\mathrm{SED}_{\mathrm{S}}$ is generally found to be statistically in good agreement with the sum of the UV and IR SFR $\left(\mathrm{SFR}_{\text {tot }}\right.$; e.g. see Wuyts et al. 2011). Furthermore, in our case the use of the

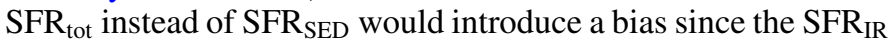
is often determined only for massive galaxies.

In Fig. 3 we plot the cumulative stellar mass distributions of the LGRB host galaxies and the star-forming galaxies of the UltraVISTA survey at $z<1$ weighted by their SFR, using the same methods as illustrated above. For the comparison with the LGRB host stellar masses, we took the stellar mass completeness of the survey at the different redshifts into account (Ilbert et al. 2013), as well as the $K$-magnitude limit. In addition to the three LGRBs excluded for the $L_{\mathrm{K}}$ comparison, we carried out the comparison by not considering the remaining LGRBs having a stellar mass below the completeness limit of the survey. A total of five LGRBs are therefore excluded (the two LGRBs not having detections or deep upper limits in the NIR are among those). For the same reason as reported in Sect. 4.1, we verified that by only considering the UltraVISTA galaxies at $0.5<z<0.8$ (always taking the stellar mass completeness of the survey into account) we obtain a similar distribution.

As already suggested by the $L_{\mathrm{K}}$ distributions, the stellar mass distribution of LGRBs is very different from what is expected from the UltraVISTA survey. Indeed, from the KolmogorovSmirnov (K-S) test, we can discard the hypothesis that the two samples are drawn from the same distribution $\left(p=9 \times 10^{-5}\right)$.

\section{Boissier's method}

Following the method of Boissier et al. (2013), we can use our sample to determine the LGRB efficiency (the LGRB rate to SFR rate, called GRB bias in Boissier et al. 2013). This 


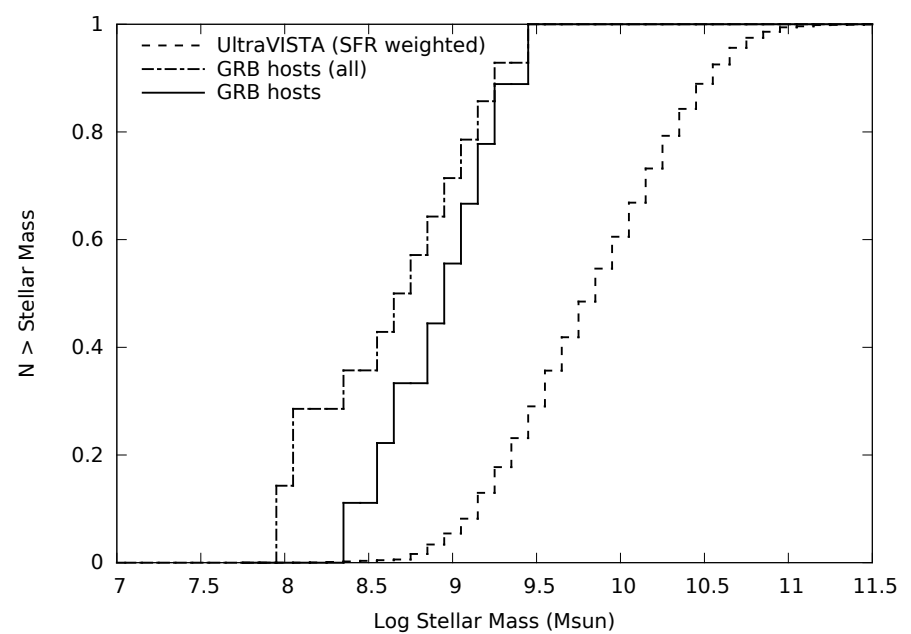

Fig. 3. Cumulative stellar mass distribution of the star-forming galaxies in the UltraVISTA survey at $z<1$ weighted by the SFR (dashed line) compared to that of the stellar masses of the LGRB hosts in our sample (dot-dashed line: whole sample; solid line: excluding the five host galaxies not complying with the completeness limits of the UltraVISTA survey).

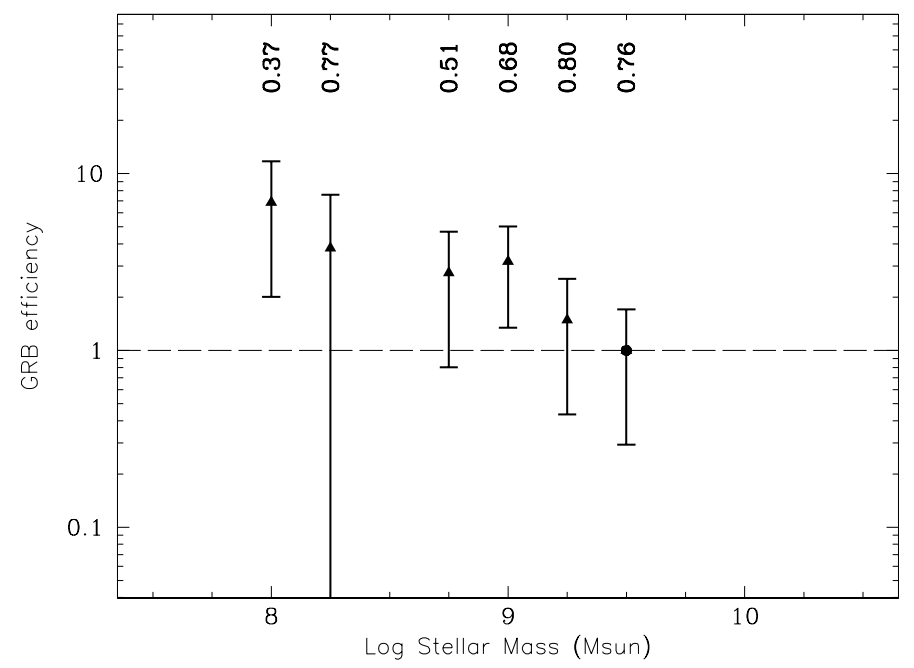

Fig. 4. LGRB efficiency (LGRB rate to SFR ratio) determined from the stellar mass distribution of our sample, following the method presented in Boissier et al. (2013). The results are normalised at $\log (M)=9.5 M_{\odot}$. The numbers above the points indicate the median redshift of the hosts contributing to the respective stellar mass bin.

method is based on comparing the stellar masses of a sample of LGRB hosts and of star-forming galaxies in the same redshift range (but lower than $\sim 1$ ). In this redshift range, the stellar mass function is nearly constant in the stellar mass range where it can be measured (above $\sim 10^{9} M_{\odot}$, e.g. Ilbert et al. 2013). To compute the efficiency, we use the same prescription for the star-forming galaxies as in Boissier et al. (2013). For the LGRB hosts, we use our sample that has the advantage of being better defined and complete with respect to the compilation used in that paper.

This method is in principle very similar to the comparison in the previous section, but includes prescriptions on star-forming galaxies allowing us to compute the variation in the efficiency with stellar mass, taking the median redshift of each bin into account. The results (see Fig. 4) have large error bars because of small statistics and are extrapolated at low masses (assuming the

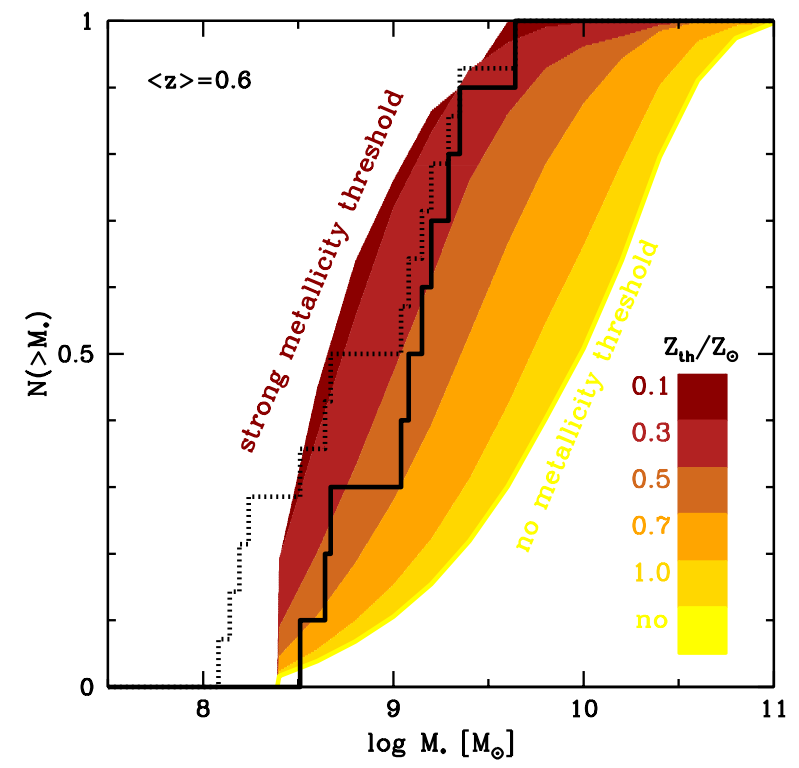

Fig. 5. Cumulative distribution of the stellar masses (including the old stellar population) of the LGRB hosts in our sample (dotted line: whole sample; solid line: with $\log M_{\star}>8.4 M_{\odot}$, to comply with the simulation limits) compared to what is obtained by LGRB host galaxy simulations. The observed distribution is explained by a metallicity cut favouring low metallicities $\left(Z<0.5 Z_{\odot}\right)$.

stellar mass function does not vary with redshift also at these masses); however, we confirm the trend towards decreasing efficiency with stellar mass, already hinted at in Boissier et al. (2013).

\section{Inferring the metallicity bias}

We can compare the stellar mass distribution of the LGRB hosts with those found from simulations. We applied the method of Campisi et al. (2009), using a galaxy catalogue constructed by combining high-resolution $N$-body simulations with a semianalytic model of galaxy formation ${ }^{11}$. The candidate host galaxies of LGRBs are selected by extracting the information for the age and metallicity of newly formed stars. To each galaxy we assigned a probability of hosting a LGRB proportional to the mass of young (i.e. with ages less than a few Myr) star particles in the simulated galaxy. This is in line with our observational and theoretical understanding of the LGRB events that indicate massive stars are progenitors of LGRBs. We also built different samples by considering different metallicity thresholds for the progenitors as suggested by some theoretical models (see Yoon \& Langer 2005, 2006; Woosley \& Heger 2006; Georgy et al. 2009): from no threshold to $Z_{\text {th }}=0.1 Z_{\odot}$. In these cases, the probability assigned to each galaxy was taken to be proportional to the mass of young star particles with a metallicity below $Z_{\mathrm{th}}$.

Since our observed sample is limited to the redshift range $z=0-1$, we have considered here the simulated galaxy population at the mean redshift of $\langle z\rangle=0.6$. We also tested that the results change only a little by considering a wider redshift range in the simulation within $z<1$. For each simulated host galaxy sample we built up the cumulative distribution of stellar masses that we compared with the observed distribution of our complete

11 The simulations are a good match to galaxy mass functions (Kitzbichler \& White 2007) and reproduce the mass-metallicity relation and the FMR (Campisi et al. 2011). 


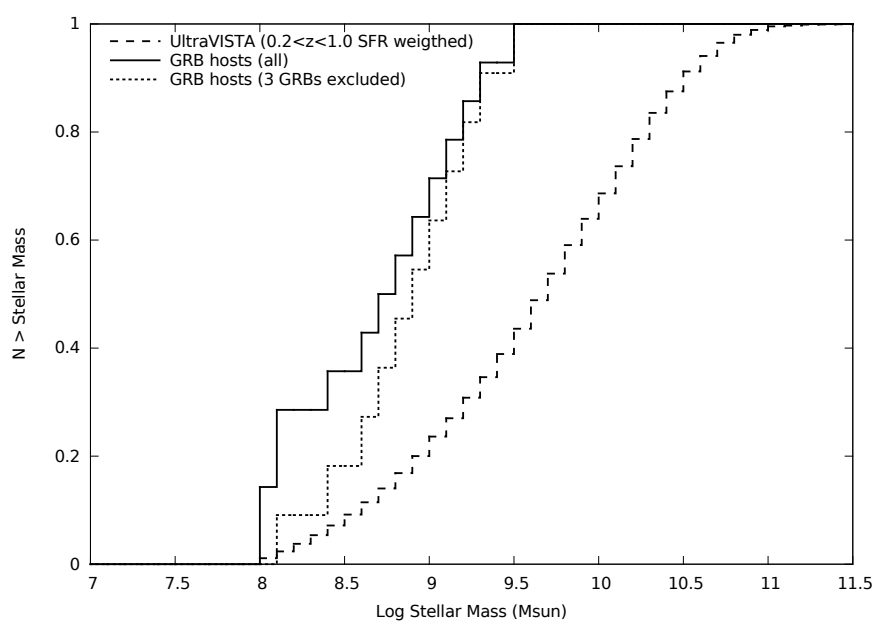

Fig. 6. Comparison between the artificially extended stellar mass cumulative distribution of the star-forming galaxies in the UltraVISTA survey at $z<1$, weighted by the SFR, (dashed line) and that of the LGRB hosts in our sample (solid line: whole sample; dotted lines: excluding three GRBs as explained in the text). Illustrated here is the slope of unity case for the SFR- $M$ relation (see text for details).

sample in Fig. 5. For this plot, we can use the stellar masses obtained by the SED fitting including the correction for old stellar population (see the end of Sect. 3). As explained above, such a correction does not change the mass distribution significantly. From the plot it is clear that the observed distribution is very far from that expected in the case of no metallicity threshold. In fact, the K-S test performed in Sect. 4.2 applies to this case. A strong metallicity threshold, i.e. $Z_{\mathrm{th}}=0.1$, predicts a distribution that is too biased towards low stellar masses. The distribution of our complete sample seems to favour a metallicity threshold $Z_{\text {th }}=0.3-0.5 Z_{\odot}$.

\section{Discussion}

Our results show that LGRB host galaxies at $z<1$ are much fainter than would be expected if GRBs were direct tracers of star formation. This result has also been confirmed by the findings of Perley et al. (2015c).

Some of the GRB host galaxies stellar masses are close to the UltraVISTA completeness limits. To check and strengthen the validity of our results, we can compare the stellar mass distribution of GRB host galaxies to an artificially extended distribution of the UltraVISTA star-forming galaxies down to $\log M_{\star}=8.0 M_{\odot}$. We proceeded as follows: (i) for each redshift bin, we considered the mass function of star-forming galaxies of Ilbert et al. (2013), and supposing no evolution of its faint end slope, we extended it to $\log M_{\star}=8.0 M_{\odot}$; (ii) to weight the SFR, we applied a SFR $-M$ relation to determine the average SFR for each stellar mass bin. The value of the slope of this relation is still being debated, and it generally varies between a slope of 0.5 and a slope of unity (e.g. Wuyts et al. 2011, and references therein). We performed our test using a slope of unity but also the more conservative case of a slope of 0.5 (Atek et al. 2014, using observations with the HST Wide Field Camera 3, report a slope of 0.65 down to $\log M_{\star}=8.0 M_{\odot}$ ). This last case would increase the weight of low-mass galaxies compared to that of a much steeper slope and therefore bring the UltraVISTA distribution closer to that of GRB host galaxies. In both cases the discrepancy between the distribution is still present (see Fig. 6). A KS test gives a null probability of $p=(0.1,1.7) \times 10^{-3}$, respectively, for the two slope values $\left(p=(0.9,7) \times 10^{-3}\right.$, without considering the two GRBs without strong limits in $\mathrm{K}$ and the one at $z<0.2$ ).
To make the two distributions consistent, a steeper faint end of the mass function would be needed, even for the 0.5 slope case. For example, considering the redshift bin $0.5<z<0.8$, the $\alpha_{2}$ index form Table 2 of Ilbert et al. 2013 should pass from $\alpha_{2}=-1.43$ to $\alpha_{2}=-1.86$ for the half case, and to $\alpha_{2}<-2.2$ for the unity case (to $\alpha_{2}<-1.63,<-2.1$, respectively, if the two GRBs without strong limits in near-infrared and the one at $z<0.2$ are excluded). To our knowledge, no survey has ever found such steep slopes at $z<1$, and those values correspond better to the slopes found at $z>2$ (e.g. Santini et al. 2012). If this condition is not fulfilled, the consistency can only be achieved if a shallower SFR- $M$ relation describes the low-mass galaxy population. For a flat SFR- $M$ relation, the two distributions would be consistent.

In our $z<1$ sample, dark LGRBs may be slightly underrepresented, even if not statistically significant, with respect to the total sample (20-30\% expected, depending on the definition; Melandri et al. Melandri et al. 2012). Under the hypothesis that dark bursts are preferably associated with massive hosts (we note nonetheless that the host of the dark LGRB in our sample is not massive: GRB100621, $\log \left(M_{\star}\right)=9.0 \pm 0.5 M_{\odot}$; see also Perley et al. 2013 for further examples), we verify that the addition of a few massive hosts would imply only a small change to the median of the stellar mass distribution, still indicating the preference for low metallicities $\left(Z<0.5 Z_{\odot}\right)$.

We furthermore checked the solidity of this result by using the strict stellar mass upper limits obtained using the Bell et al. (2003) method. The resulting metallicity threshold upper limit is $Z_{\text {th }}=0.7 Z_{\odot}$.

The redshift completeness of the BAT6 sample is 95\%. Two GRBs of the sample lack redshift information. Again, even under the strong assumption that both of them occurred in massive host galaxies at $z<1$, the change in the median of the stellar mass distribution would not be enough to significantly impact our results.

To further quantify the observed discrepancy between the GRB and field galaxy stellar mass distribution, we attempted to also perform the following test. For each GRB in the sample, we extracted a galaxy from the UltraVISTA star-forming galaxy sample randomly, following the redshift distribution of the GRBs in our sample and taking into account that the probability to find a GRB is proportional to the SFR. For each selected galaxy, we picked the corresponding stellar mass values and used them to build their stellar mass distribution. We compared this distribution with that of LGRB host galaxies (with and without taking the mass limits of the survey into account) using the KS test. We repeated this procedure one thousand times. We find that in more than $90 \%$ of the cases, we can exclude that the two distributions come from the same parent population. If we consider only the GRB host galaxies complying with the survey completeness limits, we can rule out the null hypothesis in $80 \%$ of the cases. In this last scenario, the only case for which we have a result that does not allow us to discard the inconsistency of the two distribution is under the very unlikely assumption that both the GRBs without redshift information of the BAT6 sample ( 2 over 58$)$ are at $z<1$ and occurred in high-mass galaxies. Even if the results support our conclusions, we stress that this test is not appropriate to our small dataset, because the statistics is strongly affected by the fact that we are using a small sample. Solid conclusions based on this test can only be made with much larger complete samples, which are not available today at $z<1$.

Owing to the mass-metallicity relation, low metallicity is expected considering the low masses found for LGRB hosts. The comparison with the simulations allows a more quantitative 
determination. The metallicity threshold we found does not support a theoretical model that predicts extremely low metallicities for LGRB progenitor stars but agrees with the ones requiring more moderate thresholds (e.g. Georgy et al. 2009).

We stress that our result is not at odds with the observation that some GRB host galaxies have metallicities $Z>Z_{\text {th }}$. Instead, our result should be interpreted as a preference for LGRBs at $z<1$ to explode in low-metallicity galaxies (see e.g. Modjaz et al. 2008 and Krühler et al. 2015, for consistent observational results). Moreover, the $Z_{\text {th }}$ of the simulations refers to the progenitor region.

The moderate metallicity threshold inferred by our study is also predicted by some simulations or semi-analytical models of LGRB hosts. Trenti et al. (2015) consider two channels for the LGRB production: a collapsar (hence requiring metalpoor environments) and a binary system (hence metal independent). Even if their best-fit model includes a moderate metallicity bias, at low redshift it predicts too high an efficiency for the metallicity-independent channel. The authors stress the importance of having a complete sample available to improve their results. Indeed our findings, based on the BAT6 complete sample, confirm the necessity of a higher percentage of collapsar progenitors at $z<1$.

The preference for GRBs to occur in low-mass galaxies has also been found by Perley et al. $(2015 b, c)$ for a complete sample of radio, Spitzer-observed GRB host galaxies, respectively. A metallicity dependence of the GRB efficiency would be a natural consequence of the conditions necessary to the progenitor star to produce a GRB. When taking the correlation between stellar mass and metallicity into account, it is not surprising to find that LGRBs tend to explode in low-mass galaxies. What is still not clear is whether metallicity is the only factor affecting GRB efficiency (e.g. variable IMF could also play a role). Perley et al. (2015b) suggest that an enhancement of the GRB efficiency in high specific SFR (sSFR) galaxies could also be present, therefore implying a little dependence on bulk galaxy SFR, and not necessarily indicating a dependence on metallicity.

Salvaterra et al. (2012) show that a scenario where GRBs trace the cosmic star formation and their luminosity function is constant with redshift would result in a GRB distribution that peaks at a lower redshift than observed. To recover the observed redshift distribution (keeping the luminosity function constant), cuts at very low metallicity are necessary $\left(Z_{\text {th }}<0.3 Z_{\odot}\right)$. However, this is contradicted by our results: $Z_{\text {th }}=0.3 Z_{\odot}$ is really the limit condition for which a luminosity function evolution is not necessary. For a threshold $Z_{\mathrm{th}} \sim 0.5 Z_{\odot}$, the typical burst luminosity should increase with redshift as $(1+z)^{1.3 \pm 0.6}$ (Salvaterra et al. 2012).

To speculate on the prediction of the epoch at which GRB would become direct tracers of the SFR, we can put our metallicity threshold results in the formula reported by Langer \& Norman (2006) for the number of stars born with metallicity below a specified value. Assuming that the GRB luminosity function and density do not vary with redshift, and under the hypothesis made by Langer \& Norman (2006), to obtain their Eq. (5), we find that for $Z_{\text {th }}$ in the range of $0.3-0.5$, the metallicity bias would disappear at $z \sim 4$ (see Greiner et al. 2015; Perley et al. 2015c; Krühler et al. 2015 for works on GRB has star formation tracers at $z \geq 2$ ).

\section{Conclusion and perspectives}

We compared the luminosities and stellar masses of the host galaxies of the BAT6 sample at $z<1$ to those of star-forming galaxies in the UltraVISTA survey within the same redshift range. We found that at $z<1$, LGRBs tend to avoid massive galaxies and are very powerful in selecting a population of faint star-forming galaxies, partly below the completeness limits of galaxy surveys. We studied the luminosity and stellar mass distribution and found that LGRB hosts tend to have lower luminosities and stellar masses than what was expected if LGRBs were unbiased star formation tracers, as also found in previous studies on LGRB hosts. To make the two distributions consistent, a much steeper faint end of the mass function would be required, or a shallow SFR-mass relation for the low-mass galaxy population.

The use of the BAT6 complete sample keeps these results from being affected by possible biases that could influence past results based on incomplete samples.

It is important to understand the reasons for this low LGRB efficiency. To investigate, we compared the distribution of the stellar masses of LGRB hosts with the results of simulated LGRB host galaxies with different metallicity cuts on the progenitor star. We showed that the distribution is reproduced well for a metallicity threshold of $Z_{\text {th }}=0.3-0.5 Z_{\odot}$. This can be a consequence of the particular conditions needed for the LGRB progenitor star to produce a LGRB.

The first step for future works will be to determine the metallicities and SFRs of the host galaxies of the BAT6 sample at $z<1$ from their spectra and to compare them with those found for star-forming galaxies in galaxy surveys. Because the sSFR has also been invoked as a possible bias in the LGRB-SFR efficiency, we will perform a similar study on the sSFR of the same sample of galaxy as well. Ultimately, we plan to extend these studies to a higher redshift range, in order to determine if and how the LGRB efficiency evolves.

Acknowledgements. We thank the referee for the constructive suggestions for improving the paper. We acknowledge Maria Angela Campisi for making her simulations available, Olivier Ilbert and the COSMOS team for helping with the UltraVISTA data and sharing their catalogues, Daniele Malesani and Sandra Savaglio for useful discussions. S.D.V. and E.L.F. acknowledge the UnivEarthS Labex programme at Sorbonne Paris Cité (ANR-10-LABX-0023 and ANR-11IDEX-0005-02). A.F.S. acknowledges support from grants AYA2010-22111C03-02 and AYA2013-48623-C2-2 from the Spanish Ministerio de Economia y Competitividad, and grants Prometeo 2009/064 and PrometeoII 2014/060 from the Generalitat Valenciana. This work is partially based on observations with: the ESO Telescopes at Paranal Observatory under programme ID 092.D-0305, PI: S.D. Vergani and ID 092.A-0231, PI: T. Krühler; the Gemini Observatory, acquired through the Gemini Science Archive, which is operated by the Association of Universities for Research in Astronomy, Inc., under a cooperative agreement with the NSF on behalf of the Gemini partnership: the National Science Foundation (United States), the National Research Council (Canada), CONICYT (Chile), the Australian Research Council (Australia), Ministério da Ciência, Tecnologia e Inovaçáo (Brazil) and Ministerio de Ciencia, Tecnologá e Innovación Productiva (Argentina).; GROND (http:// www . mpe.mpg.de/ jcg/GROND/); Gran Telescopio Canarias (GTC), instaled in the Spanish Observatorio del Roque de los Muchachos of the Instituto de Astrofísica de Canarias in the island of La Palma; the Nordic Optical Telescope, operated on the island of La Palma jointly by Denmark, Finland, Iceland, Norway, and Sweden, in the Spanish Observatorio del Roque de los Muchachos of the Instituto de Astrofísica de Canarias; the NASA/ESA Hubble Space Telescope, obtained from the data archive at the Space Telescope Science Institute. STScI is operated by the Association of Universities for Research in Astronomy, Inc. under NASA contract NAS 5-26555; the SDSS (Funding for SDSS-III has been provided by the Alfred P. Sloan Foundation, the Participating Institutions, the National Science Foundation, and the US Department of Energy Office of Science. The SDSS-III web site is http://www . sdss3.org/. SDSS-III is managed by the Astrophysical Research Consortium for the Participating Institutions of the SDSS-III Collaboration including the University of Arizona, the Brazilian Participation Group, Brookhaven National Laboratory, Carnegie Mellon University, University of Florida, the French Participation Group, the German Participation Group, Harvard University, the Instituto de Astrofisica de Canarias, the Michigan State/Notre Dame/JINA Participation Group, Johns Hopkins University, Lawrence Berkeley National 
Laboratory, Max Planck Institute for Astrophysics, Max Planck Institute for Extraterrestrial Physics, New Mexico State University, New York University, Ohio State University, Pennsylvania State University, University of Portsmouth, Princeton University, the Spanish Participation Group, University of Tokyo, University of Utah, Vanderbilt University, University of Virginia, University of Washington, and Yale University); the Spitzer Space Telescope, which is operated by the Jet Propulsion Laboratory, California Institute of Technology under a contract with NASA; the Italian Telescopio Nazionale Galileo (TNG) operated on the island of La Palma by the Fundación Galileo Galilei of the INAF (Istituto Nazionale di Astrofisica) at the Spanish Observatorio del Roque de los Muchachos of the Instituto de Astrofisica de Canarias. Part of the funding for GROND (both hardware as well as personnel) was generously granted from the Leibniz-Prize to Prof. G. Hasinger (DFG grant HA 1850/281). Part of the research leading to these results has received funding from the European Community's Seventh Framework Programme (FP7/2013-2016) under grant agreement number 312430 (OPTICON). This research has made use of the NASA/ IPAC Infrared Science Archive, which is operated by the Jet Propulsion Laboratory, California Institute of Technology, under contract with the National Aeronautics and Space Administration. This research has made use of the http://www. mpe.mpg. de/ jcg/grbgen.html page and of the GHostS database (www. grbhosts.org), which is partly funded by Spitzer/NASA grant RSA Agreement No. 1287913.

\section{References}

Arnouts, S., Cristiani, S., Moscardini, L., et al. 1999, MNRAS, 310, 540 Artale, M. C., Pellizza, L. J., \& Tissera, P. B. 2011, MNRAS, 415, 3417 Atek, H., Kneib, J.-P., Pacifici, C., et al. 2014, ApJ, 789, 96 Basa, S., Cuby, J. G., Savaglio, S., et al. 2012, A\&A, 542, A103

Bell, E. F., McIntosh, D. H., Katz, N., \& Weinberg, M. D. 2003, ApJS, 149, 289

Berger, E., Cowie, L. L., Kulkarni, S. R., et al. 2003, ApJ, 588, 99

Bertin, E., \& Arnouts, S. 1996, A\&AS, 117, 393

Boissier, S., Salvaterra, R., Le Floc'h, E., et al. 2013, A\&A, 557, A34

Bruzual, G., \& Charlot, S. 2003, MNRAS, 344, 1000

Calzetti, D., Armus, L., Bohlin, R. C., et al. 2000, ApJ, 533, 682

Campisi, M. A., De Lucia, G., Li, L.-X., Mao, S., \& Kang, X. 2009, MNRAS, 400,1613

Campisi, M. A., Tapparello, C., Salvaterra, R., Mannucci, F., \& Colpi, M. 2011, MNRAS, 417, 1013

Castro Cerón, J. M., Michałowski, M. J., Hjorth, J., et al. 2010, ApJ, 721, 1919 Chabrier, G. 2003, PASP, 115, 763

Chisari, N. E., Tissera, P. B., \& Pellizza, L. J. 2010, MNRAS, 408, 647

Christensen, L., Hjorth, J., \& Gorosabel, J. 2004, A\&A, 425, 913

Cucchiara, A., Levan, A. J., Fox, D. B., et al. 2011, ApJ, 736, 7

Della Valle, M., Chincarini, G., Panagia, N., et al. 2006, Nature, 444, 1050

Devillard, N. 1997, The Messenger, 87, 19

Elliott, J., Greiner, J., Khochfar, S., et al. 2012, A\&A, 539, A113

Fruchter, A. S., Levan, A. J., Strolger, L., et al. 2006, Nature, 441, 463

Georgy, C., Meynet, G., Walder, R., Folini, D., \& Maeder, A. 2009, A\&A, 502, 611

Graham, J. F., \& Fruchter, A. S. 2013, ApJ, 774, 119

Greiner, J., Bornemann, W., Clemens, C., et al. 2008, PASP, 120, 405

Greiner, J., Krühler, T., Klose, S., et al. 2011, A\&A, 526, A30

Greiner, J., Fox, D. B., Schady, P., et al. 2015, ApJ, 809, 76

Han, X. H., Hammer, F., Liang, Y. C., et al. 2010, A\&A, 514, A24

Hjorth, J., \& Bloom, J. S. 2011, Chapter 9 in Gamma-Ray Bursts, eds. C.

Kouveliotou, R. A. M. J. Wijers, \& S. E. Woosley (Cambridge University Press)

Hjorth, J., Malesani, D., Jakobsson, P., et al. 2012, ApJ, 756, 187

Hunt, L. K., Palazzi, E., Michałowski, M. J., et al. 2014, A\&A, 565, A112

Ilbert, O., Arnouts, S., McCracken, H. J., et al. 2006, A\&A, 457, 841

Ilbert, O., McCracken, H. J., Le Fèvre, O., et al. 2013, A\&A, 556, A55
Jakobsson, P., Hjorth, J., Fynbo, J. P. U., et al. 2004, ApJ, 617, L21 Jakobsson, P., Levan, A., Fynbo, J. P. U., et al. 2006, A\&A, 447, 897 Jin, Z.-P., Covino, S., Della Valle, M., et al. 2013, ApJ, 774, 114 Kitzbichler, M. G., \& White, S. D. M. 2007, MNRAS, 376, 2 Krühler, T., Greiner, J., Schady, P., et al. 2011, A\&A, 534, A108

Krühler, T., Malesani, D., Fynbo, J. P. U., et al. 2015, A\&A, in press, DOI: $10.1051 / 0004-6361 / 201425561$

Langer, N., \& Norman, C. A. 2006, ApJ, 638, L63

Le Floc'h, E., Duc, P.-A., Mirabel, I. F., et al. 2003, A\&A, 400, 499

Le Floc'h, E., Charmandaris, V., Forrest, W. J., et al. 2006, ApJ, 642, 636

Levesque, E. M., Kewley, L. J., Berger, E., \& Jabran Zahid, H. 2010, AJ, 140, 1557

Mangano, V., Holland, S. T., Malesani, D., et al. 2007, A\&A, 470, 105

Mannucci, F., Cresci, G., Maiolino, R., Marconi, A., \& Gnerucci, A. 2010, MNRAS, 408, 2115

Mannucci, F., Salvaterra, R., \& Campisi, M. A. 2011, MNRAS, 439

Mao, J. 2010, ApJ, 717, 140

McCracken, H. J., Milvang-Jensen, B., Dunlop, J., et al. 2012, A\&A, 544, A156 Melandri, A., Sbarufatti, B., D’Avanzo, P., et al. 2012, MNRAS, 421, 1265

Michałowski, M. J., Hjorth, J., Castro Cerón, J. M., \& Watson, D. 2008, ApJ, 672,817

Michałowski, M. J., Kamble, A., Hjorth, J., et al. 2012, ApJ, 755, 85

Modjaz, M., Kewley, L., Kirshner, R. P., et al. 2008, AJ, 135, 1136

Muzzin, A., Marchesini, D., Stefanon, M., et al. 2013, ApJ, 777, 18

Niino, Y., Choi, J.-H., Kobayashi, M. A. R., et al. 2011, ApJ, 726, 88

Nuza, S. E., Tissera, P. B., Pellizza, L. J., et al. 2007, MNRAS, 375, 665

Papovich, C., Dickinson, M., \& Ferguson, H. C. 2001, ApJ, 559, 620

Perley, D. A., Cenko, S. B., Bloom, J. S., et al. 2009, AJ, 138, 1690

Perley, D. A., Levan, A. J., Tanvir, N. R., et al. 2013, ApJ, 778, 128

Perley, D. A., Krühler, T., Schulze, S., et al. 2015a, ArXiv e-prints [arXiv: 1504.02482]

Perley, D. A., Perley, R. A., Hjorth, J., et al. 2015b, ApJ, 801, 102

Perley, D. A., Tanvir, N. R., Hjorth, J., et al. 2015c, ArXiv e-prints [arXiv: 1504.02479]

Pforr, J., Maraston, C., \& Tonini, C. 2012, MNRAS, 422, 3285

Prevot, M. L., Lequeux, J., Prevot, L., Maurice, E., \& Rocca-Volmerange, B. 1984, A\&A, 132, 389

Salvaterra, R., Della Valle, M., Campana, S., et al. 2009, Nature, 461, 1258

Salvaterra, R., Ferrara, A., \& Dayal, P. 2011, MNRAS, 414, 847

Salvaterra, R., Campana, S., Vergani, S. D., et al. 2012, ApJ, 749, 68

Sanders, D. B., Salvato, M., Aussel, H., et al. 2007, ApJS, 172, 86

Santini, P., Fontana, A., Grazian, A., et al. 2012, A\&A, 538, A33

Savaglio, S., Glazebrook, K., \& Le Borgne, D. 2009, ApJ, 691, 182

Schady, P., Savaglio, S., Mueller, T., et al. 2014, A\&A, 570, A52

Schlafly, E. F., \& Finkbeiner, D. P. 2011, ApJ, 737, 103

Smith, J. A., Tucker, D. L., Kent, S., et al. 2002, AJ, 123, 2121

Soderberg, A. M., Nakar, E., Cenko, S. B., et al. 2007, ApJ, 661, 982

Svensson, K. M., Levan, A. J., Tanvir, N. R., Fruchter, A. S., \& Strolger, L.-G. 2010, MNRAS, 405, 57

Symeonidis, M., Oates, S. R., de Pasquale, M., et al. 2014, MNRAS, 443, L124

Tanvir, N. R., Fox, D. B., Levan, A. J., et al. 2009, Nature, 461, 1254

Tanvir, N. R., Rol, E., Levan, A. J., et al. 2010, ApJ, 725, 625

Tanvir, N. R., Levan, A. J., Fruchter, A. S., et al. 2012, ApJ, 754, 46

Trenti, M., Perna, R., \& Jimenez, R. 2015, ApJ, 802, 103

van der Horst, A. J., Kouveliotou, C., Gehrels, N., et al. 2009, ApJ, 699, 1087

Vergani, S. D., Flores, H., Covino, S., et al. 2011, A\&A, 535, A127

Wang, F. Y., \& Dai, Z. G. 2014, ApJS, 213, 15

Woosley, S. E., \& Heger, A. 2006, ApJ, 637, 914

Wuyts, S., Förster Schreiber, N. M., Lutz, D., et al. 2011, ApJ, 738, 106

Yoon, S.-C., \& Langer, N. 2005, A\&A, 443, 643

Yoon, S.-C., \& Langer, N. 2006, in Stellar Evolution at Low Metallicity: Mass Loss, Explosions, Cosmology, eds. H. J. G. L. M. Lamers, N. Langer, T. Nugis, \& K. Annuk, ASP Conf. Ser., 353, 63 


\section{Appendix A: SED data and plots}

For each host galaxy we report the data used with a short description of the newly obtained ones. The SED fitting plots and results for each host galaxy are reported in Fig. A.1 and Table A.1, respectively.

\section{A.1. GRB 050416A host}

To build the SED of this host we used the public TNG/DOLORES $J$-band data ${ }^{12}$; programme ID: A22_TAC107 PI: D. Malesani and the photometry published by Soderberg et al. (2007), Perley et al. (2009), Hjorth et al. (2012).

\section{A.2. GRB 050525A host}

To build the SED of this host, we obtained GTC/OSIRIS data with the $g i$ filters (programme ID: GTC31-13B PI: A. Fernandez-Soto), and we used the $R$ and $K$-band photometry published by Hjorth et al. (2012), as well as the public Spitzer IRAC1and IRAC2 data obtained under programme ID: 3653, PI: P.M. Garnavich. The GTC/OSIRIS observations were obtained on the October 23, 2013 for a total exposure of $6 \times 300 \mathrm{~s}$ and $15 \times 160 \mathrm{~s}$ in $g$ and $i$ bands, respectively.

\section{A.3. GRB 060614 host}

To build the SED of this host, we used the photometry published by Della Valle et al. (2006), Mangano et al. (2007), Hjorth et al. (2012) and the public Spitzer IRAC1 data obtained under programme ID: 90062, PI: D. A. Perley.

\section{A.4. GRB 060912A host}

To build the SED of this host, we used the public TNG/DOLORES $U B V I J$-band data ${ }^{13}$; program ID: A22_TAC107 PI: D. Malesani, the $R$ and $K$-band photometry published by Hjorth et al. (2012), and the public Spitzer IRAC1 data obtained under programme ID: 90062, PI: D. A. Perley.

\section{A.5. GRB 061021 host}

To build the SED of this host, we obtained VLT/HAWK-I photometry (ID 092.D-0305, PI: S. D. Vergani). We also used the public TNG/DOLORES $B$ and $V$-band data ${ }^{14}$; program ID: A22_TAC107 PI: D. Malesani, the public Spitzer IRAC1 data obtained under programme ID: 90062, PI: D. A. Perley, and the $R$-band photometry published by Hjorth et al. (2012).

VLT/HAWK-I observations were taken between December 28, 2013 and January 7, 2014 for a total of $30 \mathrm{~min}(15 \times 120 \mathrm{~s})$ in $J$-band and $80 \mathrm{~min}(40 \times 120 \mathrm{~s})$ in $K$-band.

\section{A.6. GRB $071112 C$ host}

To build the SED of this host we obtained GTC/OSIRIS data with the $z$ filter (programme ID: GTC31-13B PI: A. Fernandez-Soto). We also used $R$-band Gemini public data (programme ID: GN-2008B-Q-112 PI: A. Levan), TNG/DOLORES I-band data (programme ID: A17_TAC9 PI:C. Guidorzi), HST F160W data (programme ID: GN-2008B-Q-112 PI: A. Levan),

\footnotetext{
12 http://ia2.oats.inaf.it/index.php/tngarchive/tng

13 http://ia2 . oats.inaf.it/index.php/tngarchive/tng

${ }^{14}$ http://ia2 . oats.inaf.it/index.php/tngarchive/tng
}

and the public Spitzer IRAC1 data obtained under programme ID: 90062, PI: D. A. Perley.

The GTC/OSIRIS observations were obtained on February 1, 2014 under clear sky conditions for a total exposure of $21 \times 100 \mathrm{~s}$ in $z$-band.

\section{A.7. GRB0 $80319 B$ host}

To build the SED of this host we used the photometry published by Tanvir et al. (2010).

\section{A.8. GRB 080430 host}

To build the SED of this host, we obtained TNG/DOLORES photometry with the $g, i, z$ filters under the OPTICON (Optical Infrared Co-ordination Network for astronomy) programme OPT13B_71 (PI S. D. Vergani). We also used the $r$-band Keck LRIS photometry kindly made available by D. A. Perley in his preliminary LGRB hosts catalogue ${ }^{15}$, the HST F160W data (programme ID: GN-2008B-Q-112 PI: A. Levan), as well as the public Spitzer IRAC1 data obtained under programme ID: 90062, PI: D. A. Perley.

The TNG observations were obtained on January 28, 2014 and February 1, 2014 for a total of $8250 \mathrm{~s}, 6900 \mathrm{~s}$, and $9630 \mathrm{~s}$, in the $g, i, z$ filters, respectively.

\section{A.9. GRB $080916 A$ host}

To build the SED of this host, we obtained VLT/HAWK-I photometry (ID 092.D-0305, PI: S. D. Vergani), we used photometry obtained by the GROND (see 2.1), the HST F160W data (programme ID: GN-2008B-Q-112 PI: A. Levan), and the public Spitzer IRAC1 data obtained under programme ID: 90062, PI: D. A. Perley.

The $K, J$-band VLT/HAWK-I observations started on November 10 and 13, 2013, respectively, under excellent seeing conditions. We obtained a total of $26 \mathrm{~min}(13 \times 120 \mathrm{~s})$ in the $J$-band and $25 \mathrm{~min}(15 \times 100 \mathrm{~s})$ in the $K$ s-band. GROND observations started on December 21, 2008. The integration of the individual $12 \mathrm{griz}$ and $360 \mathrm{JHK}$ images is of $369 \mathrm{~s}$ and $10 \mathrm{~s}$, respectively.

\section{A.10. GRB 081007 host}

To build the SED of this host, we used VLT/HAWK-I photometry (ID 092.A-0231, PI: T. Krühler), GROND photometry (see Sect. 2.1), the HST F160W data (programme ID: GN-2008B-Q112 PI: A. Levan), the photometry published in Jin et al. (2013) and the public Spitzer IRAC1 data obtained under programme ID: 80153, PI: D. A. Perley.

The HST images reveal two objects: one falling in the UVOT afterglow error circle and another separated by less than $1^{\prime \prime}$ $(<6.3 \mathrm{kpc})$. Those are not resolved by the other instruments used, and together they extend over $\sim 1.5^{\prime \prime}$. Even if it is not certain, in this paper we considered them as forming a single system.

VLT/HAWK-I observations started on October 13, 2013 for a total of $18 \mathrm{~min}(36 \times 30 \mathrm{~s})$ in $J$-band and $28 \mathrm{~min}(168 \times 10 \mathrm{~s})$ in $K$-band. GROND observations started on December 22, 2011. The integration of the individual $12 \mathrm{griz}$ and $360 \mathrm{JHK}$ images is $375 \mathrm{~s}$ and $10 \mathrm{~s}$, respectively.

15 http://www. astro.caltech.edu/LGRBhosts/ 
A\&A 581, A102 (2015)
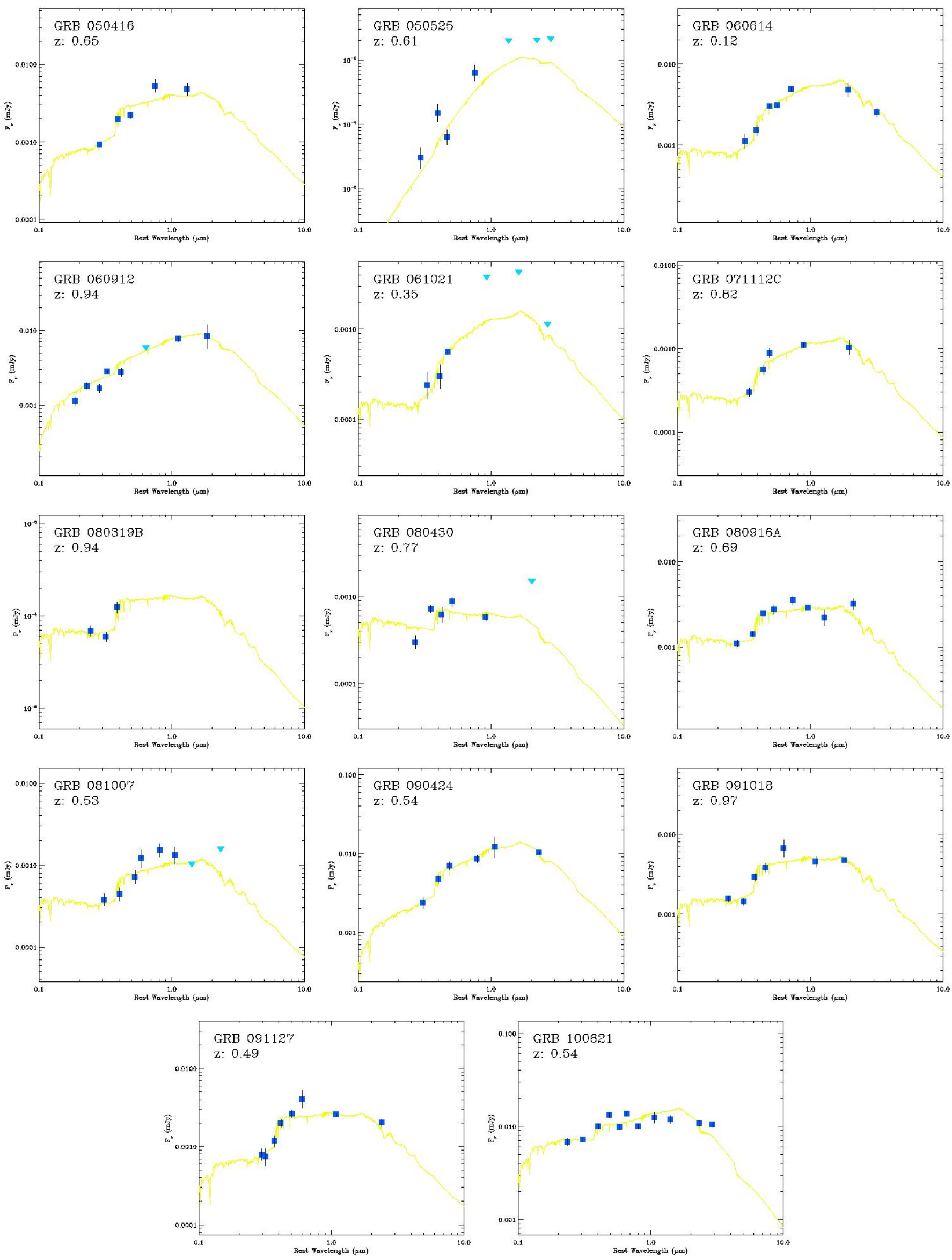

Fig. A.1. SED plots. 
Table A.1. SED fitting results.

\begin{tabular}{|c|c|c|c|c|c|c|c|c|c|c|}
\hline Host galaxy & $z$ & $\chi^{2}$ & $E_{(B-V) \text { best }}$ & $\begin{array}{c}\log \left(M_{\star}\right) \\
{\left[M_{\odot}\right]}\end{array}$ & $\begin{array}{c}\log \left(M_{\star \mathrm{inf}}\right) \\
{\left[M_{\odot}\right]}\end{array}$ & $\begin{array}{c}\log \left(M_{\star \text { sup }}\right) \\
{\left[M_{\odot}\right]}\end{array}$ & $\begin{array}{l}\text { Age } \\
{[\mathrm{yr}]}\end{array}$ & $\begin{array}{l}\log (S F R) \\
{\left[M_{\odot} \mathrm{yr}^{-1}\right]}\end{array}$ & $\begin{array}{r}\log \left(S F R_{\text {inf }}\right) \\
{\left[M_{\odot} \mathrm{yr}^{-1}\right]}\end{array}$ & $\begin{array}{r}\log \left(S F R_{\text {sup }}\right) \\
{\left[M_{\odot} \mathrm{yr}^{-1}\right]}\end{array}$ \\
\hline GRB 050416 & 0.653 & 8.3 & 0.100 & $0.910 \mathrm{E}+01$ & $0.901 \mathrm{E}+01$ & $0.923 \mathrm{E}+01$ & $0.32 \mathrm{E}+09$ & $-0.777 \mathrm{E}-01$ & $-0.418 \mathrm{E}+00$ & $0.334 \mathrm{E}+00$ \\
\hline GRB 050525 & 0.606 & 2.8 & 0.900 & $0.810 \mathrm{E}+01$ & $0.753 \mathrm{E}+01$ & $0.876 \mathrm{E}+01$ & $0.10 \mathrm{E}+08$ & $-0.284 \mathrm{E}+00$ & $-0.107 \mathrm{E}+01$ & $0.436 \mathrm{E}+00$ \\
\hline GRB 060614 & 0.125 & 2.6 & 0.000 & $0.809 \mathrm{E}+01$ & $0.792 \mathrm{E}+01$ & $0.824 \mathrm{E}+01$ & $0.50 \mathrm{E}+10$ & $-0.153 \mathrm{E}+01$ & $-0.172 \mathrm{E}+01$ & $-0.130 \mathrm{E}+01$ \\
\hline GRB 060912 & 0.937 & 6.8 & 0.000 & $0.929 \mathrm{E}+01$ & $0.917 \mathrm{E}+01$ & $0.953 \mathrm{E}+01$ & $0.14 \mathrm{E}+10$ & $0.144 \mathrm{E}+01$ & $0.997 \mathrm{E}+00$ & $0.166 \mathrm{E}+01$ \\
\hline GRB 061021 & 0.346 & 0.4 & 0.000 & $0.848 \mathrm{E}+01$ & $0.796 \mathrm{E}+01$ & $0.910 \mathrm{E}+01$ & $0.90 \mathrm{E}+10$ & $-0.391 \mathrm{E}+00$ & $-0.110 \mathrm{E}+01$ & $0.422 \mathrm{E}+00$ \\
\hline GRB 071112 & 0.823 & 1.8 & 0.000 & $0.889 \mathrm{E}+01$ & $0.871 \mathrm{E}+01$ & $0.904 \mathrm{E}+01$ & $0.55 \mathrm{E}+10$ & $-0.125 \mathrm{E}+00$ & $-0.465 \mathrm{E}+00$ & $0.336 \mathrm{E}+00$ \\
\hline GRB 080319 & 0.937 & undef & 0.000 & $0.812 \mathrm{E}+01$ & $0.776 \mathrm{E}+01$ & $0.851 \mathrm{E}+01$ & $0.57 \mathrm{E}+09$ & $-0.516 \mathrm{E}+00$ & $-0.881 \mathrm{E}+00$ & $0.263 \mathrm{E}-01$ \\
\hline GRB 080430 & 0.767 & 9.7 & 0.000 & $0.815 \mathrm{E}+01$ & $0.796 \mathrm{E}+01$ & $0.827 \mathrm{E}+01$ & $0.18 \mathrm{E}+09$ & $-0.580 \mathrm{E}-01$ & $-0.281 \mathrm{E}+00$ & $0.373 E+00$ \\
\hline GRB 080916 & 0.689 & 1.9 & 0.000 & $0.898 \mathrm{E}+01$ & $0.890 \mathrm{E}+01$ & $0.905 \mathrm{E}+01$ & $0.10 \mathrm{E}+10$ & $0.181 \mathrm{E}+00$ & $-0.123 \mathrm{E}-01$ & $0.372 \mathrm{E}+00$ \\
\hline GRB 081007 & 0.529 & 2.0 & 0.000 & $0.878 \mathrm{E}+01$ & $0.833 \mathrm{E}+01$ & $0.925 \mathrm{E}+01$ & $0.26 \mathrm{E}+10$ & $0.149 \mathrm{E}+00$ & $-0.530 \mathrm{E}+00$ & $0.753 \mathrm{E}+00$ \\
\hline GRB 090424 & 0.544 & 0.3 & 0.250 & $0.938 \mathrm{E}+01$ & $0.919 \mathrm{E}+01$ & $0.955 \mathrm{E}+01$ & $0.18 \mathrm{E}+09$ & $0.913 \mathrm{E}+00$ & $0.555 \mathrm{E}+00$ & $0.126 \mathrm{E}+01$ \\
\hline GRB 091018 & 0.971 & 1.6 & 0.000 & $0.952 \mathrm{E}+01$ & $0.942 E+01$ & $0.960 \mathrm{E}+01$ & $0.72 \mathrm{E}+09$ & $0.638 \mathrm{E}+00$ & $0.413 E+00$ & $0.882 \mathrm{E}+00$ \\
\hline GRB 091127 & 0.490 & 1.3 & 0.000 & $0.867 \mathrm{E}+01$ & $0.860 \mathrm{E}+01$ & $0.874 \mathrm{E}+01$ & $0.40 \mathrm{E}+09$ & $-0.421 \mathrm{E}+00$ & $-0.847 \mathrm{E}+00$ & $-0.962 \mathrm{E}-01$ \\
\hline GRB 100621 & 0.542 & 8.8 & 0.200 & $0.904 \mathrm{E}+01$ & $0.899 \mathrm{E}+01$ & $0.910 \mathrm{E}+01$ & $0.40 \mathrm{E}+08$ & $0.141 \mathrm{E}+01$ & $0.133 \mathrm{E}+01$ & $0.150 \mathrm{E}+01$ \\
\hline
\end{tabular}

\section{A.11. GRB 090924 host}

To build the SED of this host, we used the photometry published in Jin et al. (2013), that available from the $\operatorname{SDSS}^{16}$ and the public Spitzer IRAC1 data obtained under programme ID: 90062, PI: D. A. Perley.

\section{A.12. GRB 091018 host}

To build the SED of this host, we obtained VLT/HAWK-I photometry (ID 092.D-0305, PI: S. D. Vergani), we used the photometry obtained by the GROND (see Sect. 2.1) and the public Spitzer IRAC1 data obtained under programme ID: 90062, PI: D. A. Perley.

VLT/HAWK-I observations started on November 13, 2013 under excellent seeing conditions for a total of $40 \mathrm{~min}$
$(20 \times 120$ s $)$ in $K$-band. GROND observations started on August 30, 2011. The integration of the individual $16 \mathrm{griz}$ and $480 \mathrm{JHK}$ images is $375 \mathrm{~s}$ and $10 \mathrm{~s}$, respectively.

\section{A.13. GRB 091127 host}

To build the SED of this host, we used the photometry published in Vergani et al. (2011) and the public Spitzer IRAC1 data obtained under programme ID: 90062, PI: D. A. Perley.

\section{A.14. GRB 100621A host}

To build the SED of this host, we used the photometry published in Krühler et al. (2011) and the public Spitzer IRAC1 and IRAC2 data obtained under programme ID: 70036, PI: D. A. Perley.

${ }^{16}$ https://www.sdss3.org/dr10/imaging/ 